\title{
ANALYSIS OF A CROSS-CHANNEL MICROMIXER THROUGH THE DYNAMICS OF TRACER GRADIENT
}

\author{
Michel GonZalez \\ CNRS, UMR 6614/CORIA, Site Universitaire du Madrillet, Saint-Etienne du Rouvray, France \\ e-mail: michel.gonzalez@coria.fr
}

\begin{abstract}
The study aims at showing how the dynamics of tracer gradient accounts for the stirring mechanism produced by a periodically forced flow relevant to practical mixing operation. The numerical simulation uses the equations for the orientation and norm of the tracer gradient and an analytic model for velocity. To a large extent, the micromixer properties over different ranges of the Strouhal number are explained through the response of the tracer gradient orientation to the tilting of strain principal axes resulting from flow forcing. The analysis also reveals a rich picture of stirring as the Strouhal number is varied.
\end{abstract}

Keywords: Low-Reynolds number flows, mixing enhancement, micromixers

\section{Introduction}

In fluid flows used for mixing, stretching caused by stirring raises the contact areas and draws fluid portions to be mixed together, which hastens the molecular diffusion flux. Low-Reynolds number flows, by contrast to turbulent flows, do not start the stretching-driven cascade bringing about the shrinking of scales down to the diffusive lengthscale. Mixing of a weakly diffusive material, then, may need significant time - or distance - to be achieved. Efficient mixing has thus to be controlled, which needs precise knowledge of the stirring properties of the flow. This question is crucial in microfluidics. Chemical, biological and medical applications, in particular, have given rise to significant investigation in this field both on the practical and academic levels (Nguyen and Wu, 2005; Capretto et al., 2011; Lee et al., 2011).

The gradient of a scalar - heat, contaminant... - is the finest level at which the problem of mixing can be tackled, for it gives a direct insight into the stirring mechanisms. This approach is also plainly suited to the physics of mixing. As the gradient direction and magnitude respectively correspond to the striation orientation and thickness - fine structures meaning large gradients, the tracer gradient features are indeed closely connected to the structure of mixing patterns. The growth rate of the tracer gradient shows the conditions in which stirring may enhance the diffusion fluxes and is thus a key quantity. Although strain intensity matters, the alignment of the tracer gradient within the strain eigenframe may be the determining factor. The role of alignment may be especially critical in non-stationary regimes in which the dynamics of the tracer gradient - through its response to unsteady mechanical action - drives the growth rate. Previous studies addressed the dynamics of the scalar gradient orientation (Lapeyre et al., 2001; Garcia et al., 2005) and its role in the mechanism of the gradient growth was pointed out (Garcia et al., 2008; Gonzalez and Paranthoën, 2010).

The present study is focused on the kinematics of a tracer gradient in a periodically forced cross-channel micromixer. The purpose is twofold: i) addressing the role of the tracer gradient dynamics in stirring produced by a practical mixing device; ii) assessing the relevance of the tracer gradient approach to the analysis of stirring in this kind of device. It seems that the operation of such a micromixer has not been studied through the response of a vector field yet. 
The device is an active mixer in which the material flowing in the main channel is stirred by forcing a pulsating flow in the side channels, a standard geometry in microfluidics. Previous works (Niu and Lee, 2003; Tabeling et al., 2004; Lee et al., 2007) addressed the conditions in which chaotic advection is triggered and enhances mixing in this system. In this basic geometry, stirring properties are ruled by two parameters, namely the Strouhal number St, and the velocity ratio $\alpha$ - the ratio of maximum pulsating velocity to the maximum velocity in the main channel. Okkels and Tabeling (2004) and Lee et al. (2007) analysed the folding rate of interface segments and the mean finite-time Lyapunov exponent, respectively, by spanning both St and $\alpha$.

In this work, St is varied for two values of $\alpha$. The flowfield is simulated by the kinematic model of Niu and Lee (2003) and the Lagrangian, diffusionless equation for the tracer gradient is solved in terms of orientation and norm. The stirring properties of the flow are scrutinised through the mean growth rate of the tracer gradient norm for small to large Strouhal numbers.

\section{Cross-channel micromixer model}

A detailed description of a practical, periodically forced cross-channel micromixer was given by Lee et al. (2007). A simple sketch of the device is shown in Fig. 1.

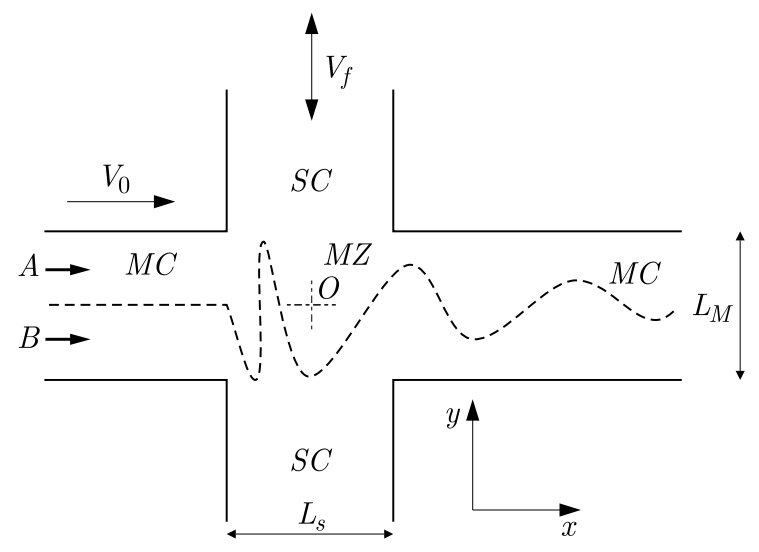

Fig. 1. Sketch of the cross-channel micromixer; MC - main channel, SC - side channel, $\mathrm{MZ}$ - mixing zone

The velocity field is simulated by the kinematic two-dimensional model proposed by Niu and Lee (2003). The flowfield is splitted over different parts of the micromixer and, assuming very low Reynolds number, the velocity field in the mixing zone is approximated as the linear combination of the velocity fields in the main and side channels. The latter are assumed to be parabolic, the velocity is steady in the main channel and periodic in the side channel. The flowfield model is expressed as follows:

- main channel

$$
u=V_{0}\left[1-\left(\frac{2 y}{L_{M}}\right)^{2}\right] \quad v=0
$$

— side channel

$$
u=0 \quad v=V_{f}\left[1-\left(\frac{2 x}{L_{S}}\right)^{2}\right] \cos \omega_{f} t
$$

— mixing zone

$$
u=V_{0}\left[1-\left(\frac{2 y}{L_{M}}\right)^{2}\right] \quad v=V_{f}\left[1-\left(\frac{2 x}{L_{S}}\right)^{2}\right] \cos \omega_{f} t
$$


This model was shown to be consistent with experimental data - including the chaotic regime - (Lee et al., 2007).

It is straightforward to derive the strain rate, $\sigma=\sqrt{\sigma_{n}^{2}+\sigma_{s}^{2}}-$ with $\sigma_{n}=\partial u / \partial x-\partial v / \partial y$ and $\sigma_{s}=\partial u / \partial y+\partial v / \partial x$ being the normal and shear strain components - and the vorticity, $\omega=\partial v / \partial x-\partial u / \partial y$ from Eqs. (2.1)-(2.3). The orientation of the strain principal axes in the fixed frame of reference $(x, y)$ is defined by the angle $\Phi$ through $\tan (2 \Phi)=\sigma_{n} / \sigma_{s}$. As $\sigma_{n}=0$ in the whole flowfield, the strain rate reduces to $\sigma=\left|\sigma_{s}\right|$. The orientation of strain principal axes is a simple piecewise function defined as

$$
\Phi=\left\{\begin{array}{lll}
0 & \text { for } & \sigma_{s}>0 \\
\pi / 2 & \text { for } & \sigma_{s}<0
\end{array}\right.
$$

The local structure of the flow is defined by $Q=\sigma^{2}-\omega^{2}(Q>0$ in hyperbolic regions and $Q<0$ in elliptic regions; Okubo, 1970; Weiss, 1991). The flow structure in the side channel is a pure shear and $Q=0$ in this part of the micromixer. In the mixing zone, where $Q \sim x y \cos \omega_{f} t$, the Eulerian local structure is periodic in time as already mentioned by Lee et al. (2007). However, the evolution of $Q$ along Lagrangian trajectories is of course more complex.

\section{Tracer gradient equations}

The general equation for the gradient $\mathbf{G}$ of a diffusionless scalar - or tracer - is

$$
\frac{D \mathbf{G}}{D t}=-\mathbf{A}^{\mathrm{T}} \mathbf{G}
$$

where $\mathbf{A}=\nabla \mathbf{u}$ is the velocity gradient tensor. With $\mathbf{G}=|\mathbf{G}|(\cos \theta, \sin \theta)$, Eq. (3.1) gives the Lagrangian equations for the orientation and norm of $\mathbf{G}$ (Lapeyre et al., 1999)

$$
\frac{D \theta}{D t}=\frac{1}{2}\{\omega-\sigma \cos [2(\theta+\Phi)]\} \quad \frac{2}{|\mathbf{G}|} \frac{D|\mathbf{G}|}{D t}=-\sigma \sin [2(\theta+\Phi)]
$$

The growth rate, $\eta=(1 /|\mathbf{G}|)(D|\mathbf{G}| / D t)$, takes the minimum value $-\sigma / 2$ for $\mathbf{G}$ aligning with the extensional strain direction defined by $\theta_{e}=-\Phi+\pi / 4$ and reaches the maximum value $\sigma / 2$, when $\mathbf{G}$ aligns with the compressive strain direction given by $\theta_{c}=-\Phi-\pi / 4$.

As a result of the alternating behaviour of $\Phi$, Eq. (2.4), the tracer gradient experiences unsteady forcing through its orientation with respect to the strain principal axes. As is shown in this study, the variations of $\Phi$ are actually essential to explain the mean growth rate of the tracer gradient in function of the flow properties.

The Lagrangian dynamics of the tracer gradient orientation is derived by solving Eq. $(3.2)_{1}$ together with the tracking of particles given by $D \mathbf{x} / D t=\mathbf{u}$ with $\mathbf{x}$ being the position vector and $\mathbf{u}$ the modelled velocity field in the mixer. The growth rate of the tracer gradient is then directly given by Eq. (3.2) 2 without solving for the gradient norm. High growth rate indicates good stirring properties through fast enhancement of local gradients.

The numerical method is a fourth-order Runge-Kutta scheme. Initially, 1000 particles are located on a line at $y=0$ from $x=-L_{S} / 2$ to $x=L_{S} / 2$. As we are strictly interested in the properties of a single mixing cavity - and not a series of elemental micromixers, each particle leaving the computational domain at $x=L_{S} / 2$ is reinjected at $(x, y)=\left(-L_{S} / 2,0\right)$ with the initial condition $\theta=\pi / 2$ for the orientation of the tracer gradient, which mimics the tracking of an interface between two different scalar quantities - say, $A$ and $B$ as shown in Fig. 1. The numerical timestep is $\Delta t=T_{f} / 3200$ with $T_{f}=2 \pi / \omega_{f}$ being the time period of the flow forcing. Statistics are derived by averaging over all the instantaneous values computed along the 
Lagrangian paths during a time interval $T=200 T_{f}$. As $T$ amounts to several mean residence time - 50 at least - this is enough to account for the differences in residence time over the particle ensemble. Averaging over the values recorded on Lagrangian trajectories is denoted by brackets. Those averaged quantities duly include Lagrangian information on the evolution of the tracer gradient. Integrating along trajectories - for the strain and growth rates, for instance strictly shows the same behaviour.

\section{General features of the kinematics of tracer gradient}

The stirring properties of the cross-channel device are described by the amplitude parameter, $\alpha=V_{f} / V_{0}$ and the Strouhal number St $=L_{S} / V_{0} T_{f}$ (Okkels and Tabeling, 2004; Lee et al., 2007). In this study, the influence of the Strouhal number is analysed for $\alpha=1$ and $\alpha=3$ with $L_{M}=L_{S}=1$.

The Lagrangian evolution of the tracer gradient tightly depends on the residence time inside the micromixer cavity. The mean residence time $T_{r}$ of a particle injected at $\left(-L_{S} / 2,0\right)$ and leaving the mixing zone at section $x=L_{S} / 2$ is shown in Fig. 2. For a given value of the Strouhal number, the residence time in the side channels - and thus $T_{r}$ - obviously grows with $\alpha$. At large Strouhal numbers, the time spent in the side channels tends to zero and $T_{r}$ approaches the convection timescale $T_{c}=L_{S} / V_{0}$ as expected. The ratio of the residence time to the time period of the flow forcing $T_{r} / T_{f}=\mathrm{St} T_{r} / T_{c}$, thus varies as St at large St values, as shown by the plot of $T_{r} / T_{f}$.
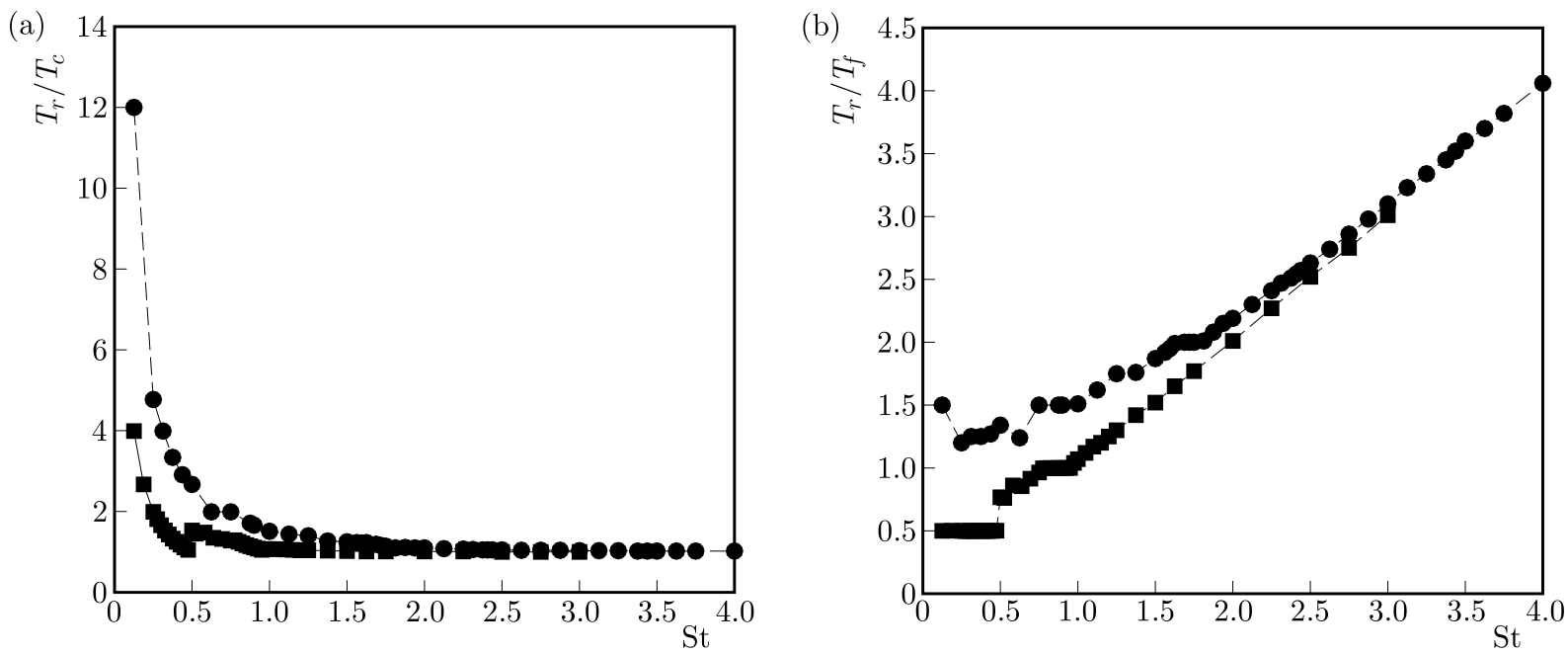

Fig. 2. Mean residence time in the micromixer cavity; (a) $T_{r} / T_{c}$, with $T_{c}=L_{S} / V_{0}$ being the convection timescale, (b) $T_{r} / T_{f}$, with $T_{f}$ being the time period of the flow forcing; squares: $\alpha=1$, circles: $\alpha=3$

Overall, the evolution of the tracer gradient derived from the tracking of particles injected at $\left(-L_{S} / 2,0\right)$ is dominated by rotation. At small Strouhal numbers, however, they are more likely to experience a pure shear regime as a result of significant time spent in the side channels. This behaviour is shown by the plots of the respective strain $(Q>0)$, rotation $(Q<0)$ and shear $(Q=0)$ events ratios along the Lagrangian paths (Fig. 3).

The dependence of the tracer gradient dynamics upon the Strouhal number in terms of the orientation and norm growth rate reveals a rich phenomenology. From the plot of $\langle\eta\rangle /\langle\sigma\rangle-$ which gives a measure of the efficiency of the micromixer (Fig. 4), Strouhal number ranges promoting good stirring are found together with narrow ranges where stirring is certainly quite poor. While 

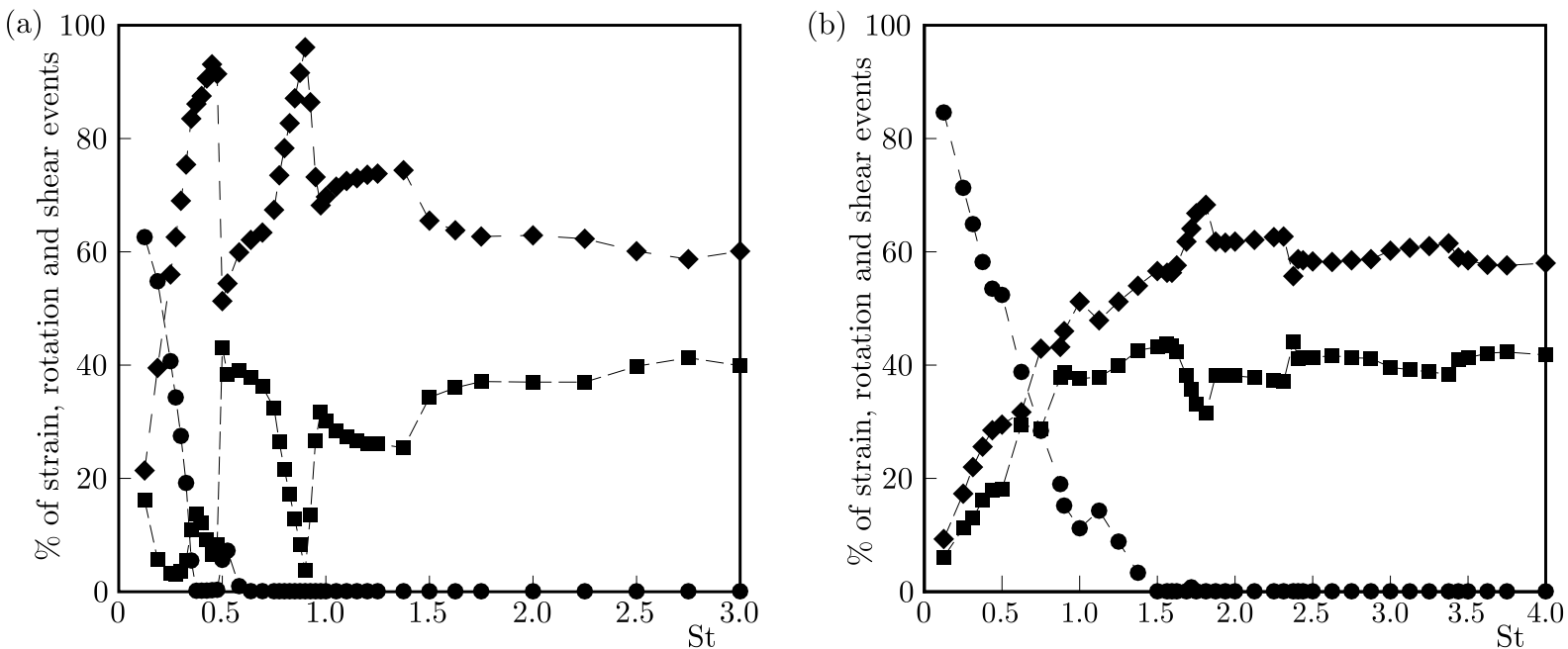

Fig. 3. Ratios of strain-, rotation- and shear-dominated events along the Lagrangian paths vs. Strouhal number; (a) $\alpha=1$, (b) $\alpha=3$; squares: strain, diamonds: rotation, circles: shear

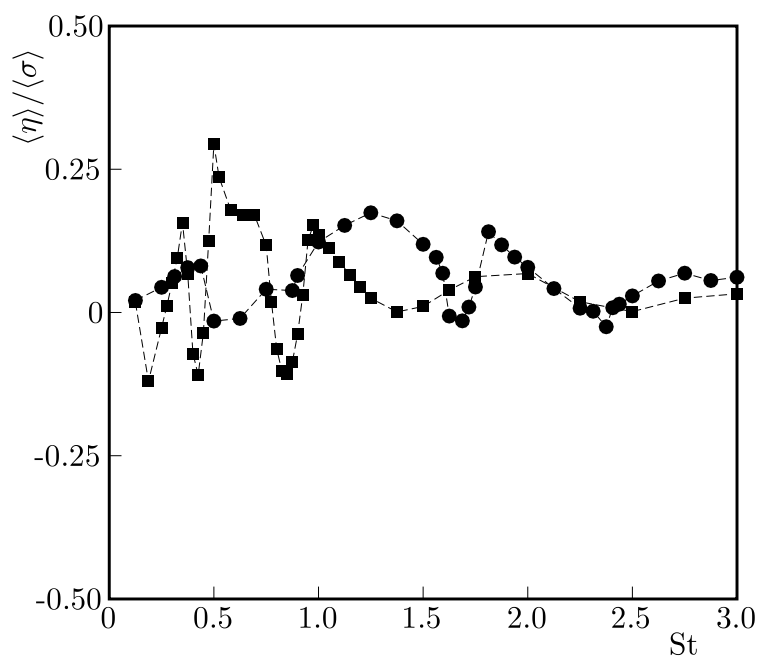

Fig. 4. Ratio of the mean growth rate to the mean rate of strain, $\langle\eta\rangle /\langle\sigma\rangle$, vs. Strouhal number; squares: $\alpha=1$, circles: $\alpha=3$

its theoretical maximum value is $0.5,\langle\eta\rangle /\langle\sigma\rangle$ reaches 0.29 for $\alpha=1$ and 0.17 , at best, for $\alpha=3$. This is due to a moderate statistical alignment of the tracer gradient with the compressional strain which most likely results from overall prevailing rotation.

The rather weak alignment of $\mathbf{G}$ with compressional strain is confirmed by Fig. 5 displaying $\langle\sigma\rangle,\langle-\sin \zeta\rangle-$ with $\zeta=2(\theta+\Phi)-$ and $\langle\eta\rangle$ in function of the Strouhal number. Far from unity the value reached when $\mathbf{G}$ is parallel to compression, the maximum for $\langle-\sin \zeta\rangle$ is 0.31 for $\alpha=1$ and 0.16 for $\alpha=3$. The mean growth rate depends on the strain level, but the dependence on the tracer gradient orientation through $\sin \zeta$ is stronger. This is clear beyond $\mathrm{St} \simeq 0.7$ for $\alpha=1$ and $\mathrm{St} \simeq 1$ for $\alpha=3$. These results thus suggest that the stirring properties of the flow can be mainly explained in terms of the dynamics of tracer gradient orientation. As the tracer gradient is locally normal to the interface separating the material to be mixed, this view agrees with analyses based on the deformation of interface segments (Okkels and Tabeling, 2004; Tabeling et al., 2004). 
(a)
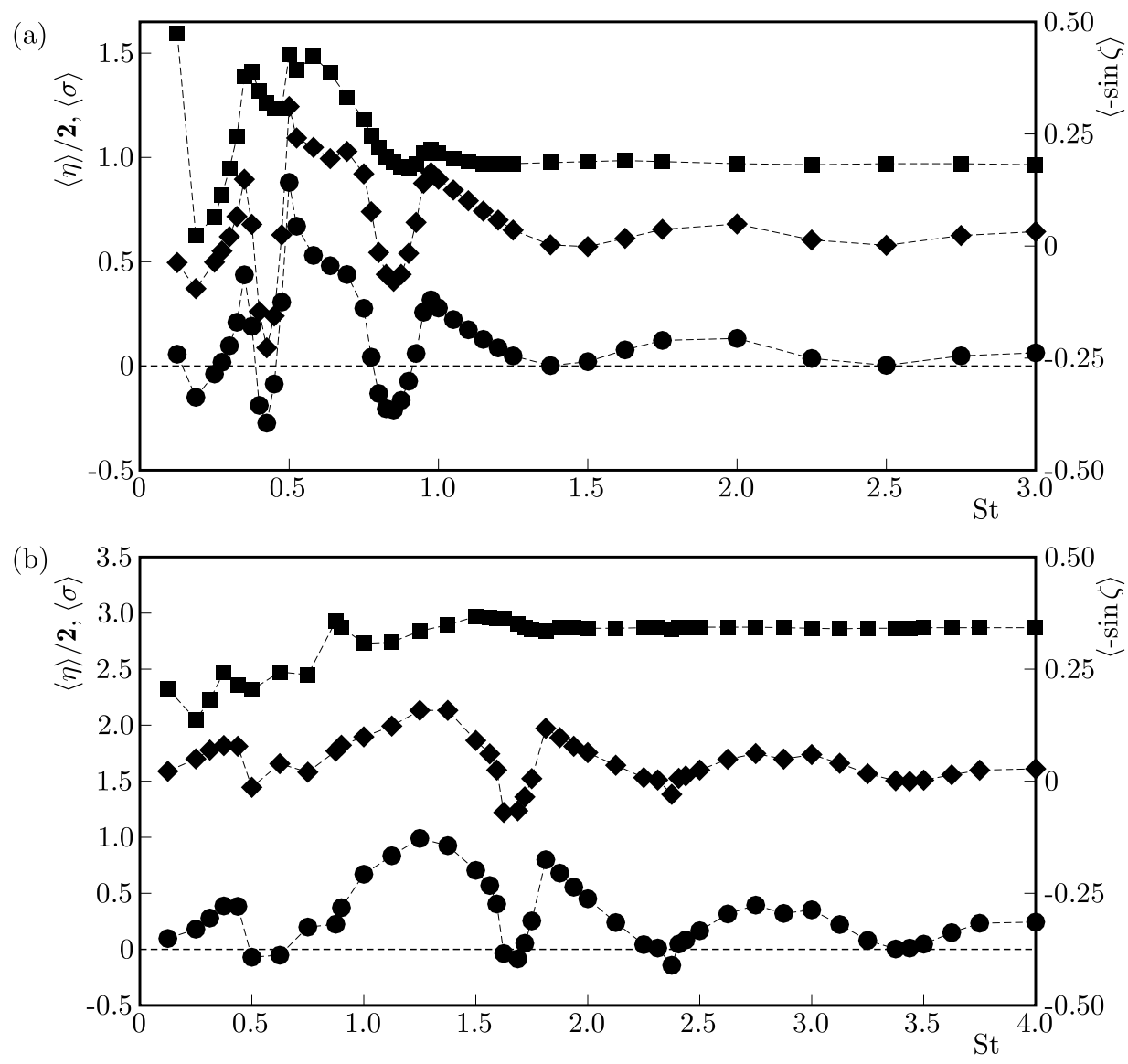

Fig. 5. Mean rate of strain $\langle\sigma\rangle$ statistical alignment given by $\langle-\sin \zeta\rangle$ and mean growth rate $\langle\eta\rangle$ vs.

Strouhal number; (a) $\alpha=1$, (b) $\alpha=3$; squares: $\langle\sigma\rangle / 2$, diamonds: $\langle-\sin \zeta\rangle$, circles: $\langle\eta\rangle$

\section{Dependence of stirring properties on the Strouhal number}

\subsection{Small Strouhal number}

Figure 6 confirms that the mean residence time inside the side channels is significant at small Strouhal numbers. As a result, the statistics of tracer gradient orientation in this range of the Strouhal number is governed by pure shear $(Q=0)$.

From Eq. (2.2), the transversal velocity $v$ and the shear component of strain $\sigma_{s}$ are in phase in the side channels, where Lagrangian particles move with $x=$ const. Each turning back of a Lagrangian particle in the side channel thus coincides with a $\pi / 2$-tilting of the strain principal axes, Eq. (2.4), that makes $\sin \zeta$ - and hence $\eta$ - change sign; the way back is always covered with the sign reversed for the growth rate which, on average, is therefore close to zero in the side channels. Figure 7 shows the trajectories as well as the Lagrangian histories of $\sigma$, $\sin \zeta$ and $\eta$ for $\alpha=1$ and $\mathrm{St}=0.125$.

The mean values conditioned on pure shear $\langle-\sin \zeta \mid Q=0\rangle$ and $\langle\eta \mid Q=0\rangle$, are close to zero up to St $\simeq 0.35$ (Fig. 8a). The same behaviour for $\alpha=3$ is displayed in Fig. 8 b.

\subsection{Large Strouhal number}

The behaviour of the tracer gradient at large Strouhal numbers is governed by the tilting of strain principal axes in the mixing zone. The tilting of strain principal axes occurs in the elliptic regions; it is easy to show, from the expressions for $\sigma_{s}$ and $Q$, that $\sigma_{s}$ changes sign which also amounts to $\sigma=0$ - only if $Q<0$. As St is increased, the mean tilting frequency $f_{\Phi}$ 


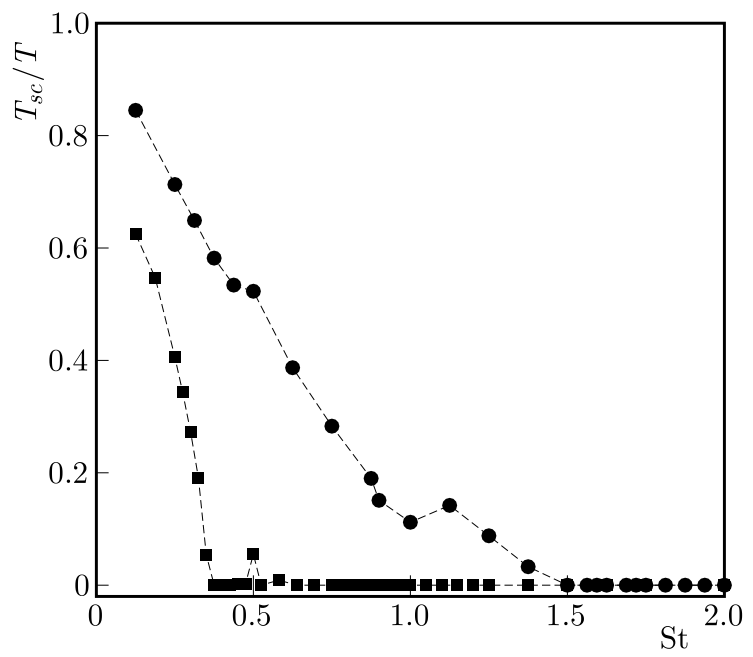

Fig. 6. Mean ratio $T_{S C} / T$ of time spent in the side channels during simulation; squares: $\alpha=1$; circles: $\alpha=3$

(a)

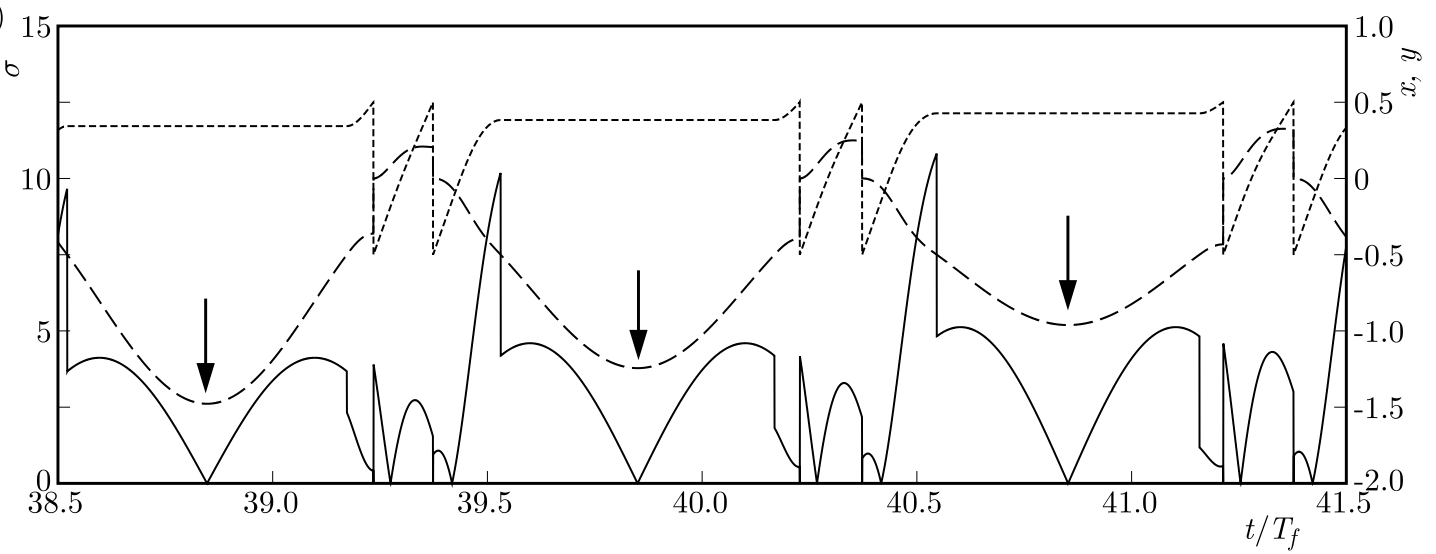

(b)

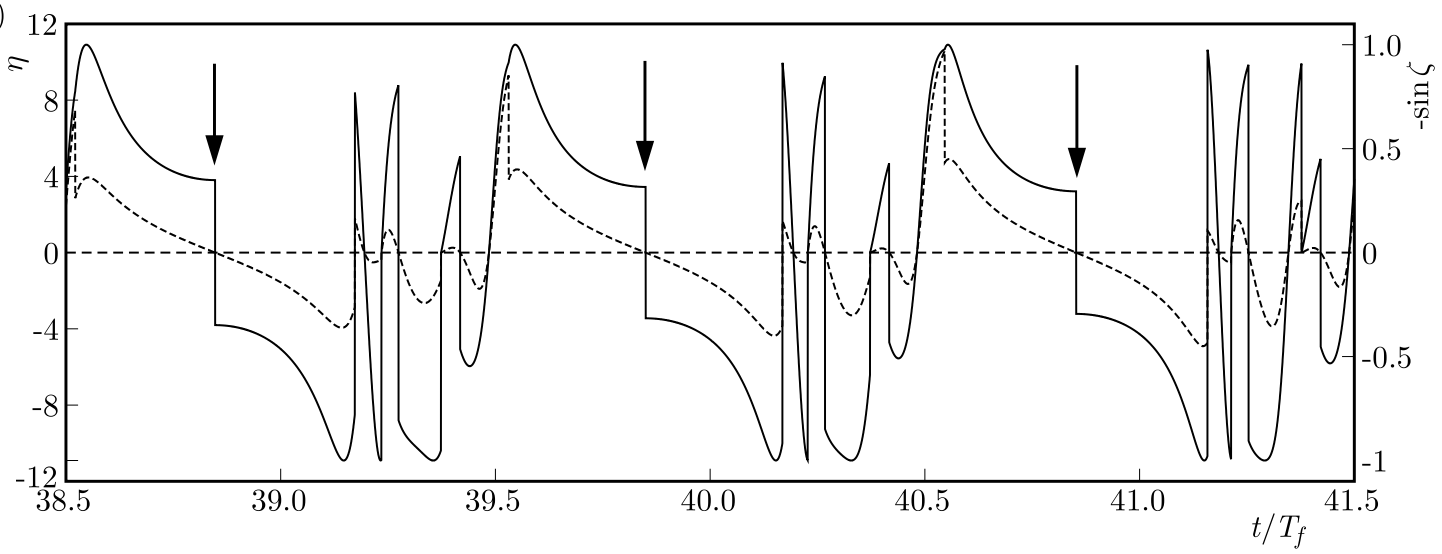

Fig. 7. Lagrangian signals for $\alpha=1$ and St $=0.125$; (a) solid line: $\sigma$, short dash: $x$, long dash: $y$; (b) solid line: $-\sin \zeta$, dashed line: $\eta$; each arrow shows a turning back in a side channel

grows linearly as shown in Fig. 9 ( $T_{r} \simeq T_{c}$ at large Strouhal numbers, Fig. 2). At large Strouhal numbers, the tracer gradient thus experiences faster and faster changes in the strain direction. The latter become too fast for the tracer gradient to respond and its orientation gets closer and closer to the direction of a bisector of strain principal axes for which $\sin \zeta=0$ - and $\eta=0$. As a result, the mean growth rate decays at large Strouhal numbers. 
(a)

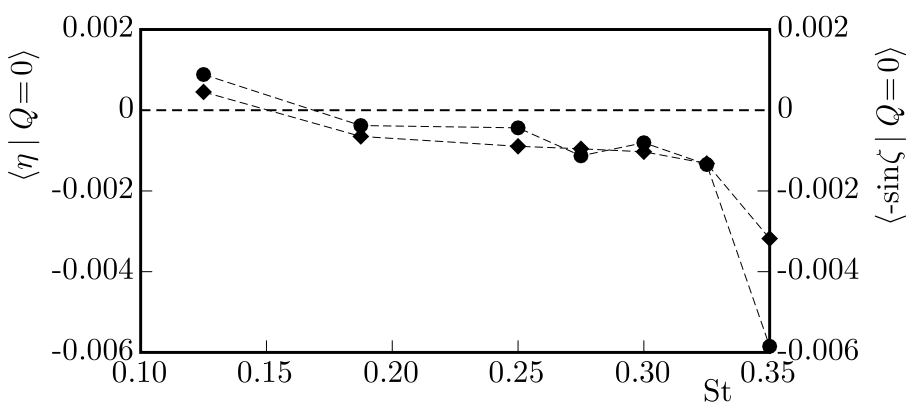

(b)

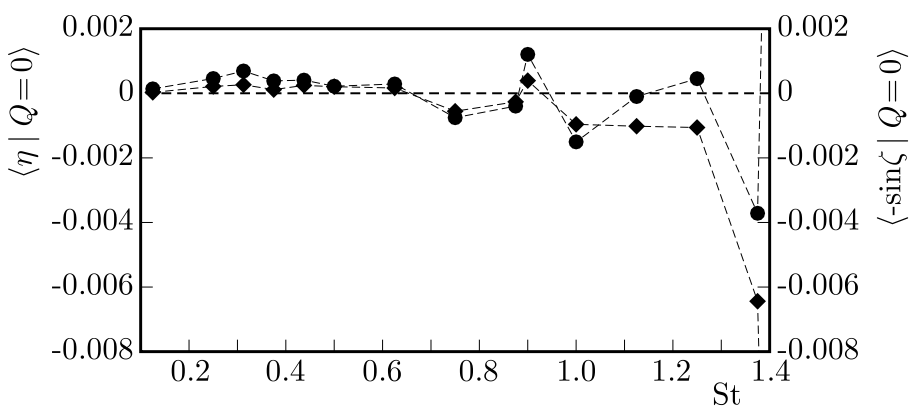

Fig. 8. Mean values of $-\sin \zeta$ and $\eta$ conditioned on pure shear vs. Strouhal number; (a) $\alpha=1$,

(b) $\alpha=3$; diamonds: $\langle-\sin \zeta \mid Q=0\rangle$, circles: $\langle\eta \mid Q=0\rangle$

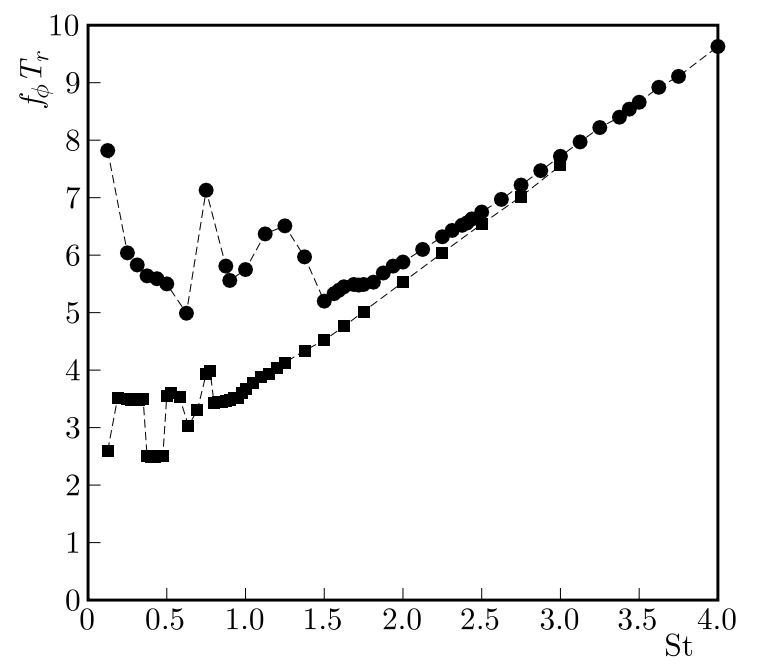

Fig. 9. Mean tilting frequency of strain principal axes normalised by the mean residence time in the mixing cavity vs. Strouhal number; squares: $\alpha=1$; circles: $\alpha=3$

On a more general level, this mechanism is clearly reminiscent of the nonadiabatic regime of the scalar gradient response to unsteady forcing in which the scalar gradient does not keep up with fast changes in strain persistence - or in strain principal axes direction (Garcia et al., 2008; Gonzalez and Paranthoën, 2010). The probability density function (p.d.f) of the tracer gradient orientation (Fig. 10) shows the gradual preferential alignment with a bisector of strain principal axes at large Strouhal numbers.

For $\alpha=1$, the variance of $\sin \zeta$ is $0.243,0.102$ and 0.0707 at $\mathrm{St}=2,3$ and 4, respectively; for $\alpha=3$ these values are $0.687,0.548$ and 0.396 . The tendency is less marked when $\alpha=3$ for which higher strain and vorticity levels result in a better response of the tracer gradient to the mechanical action of the velocity gradient. 
(a)

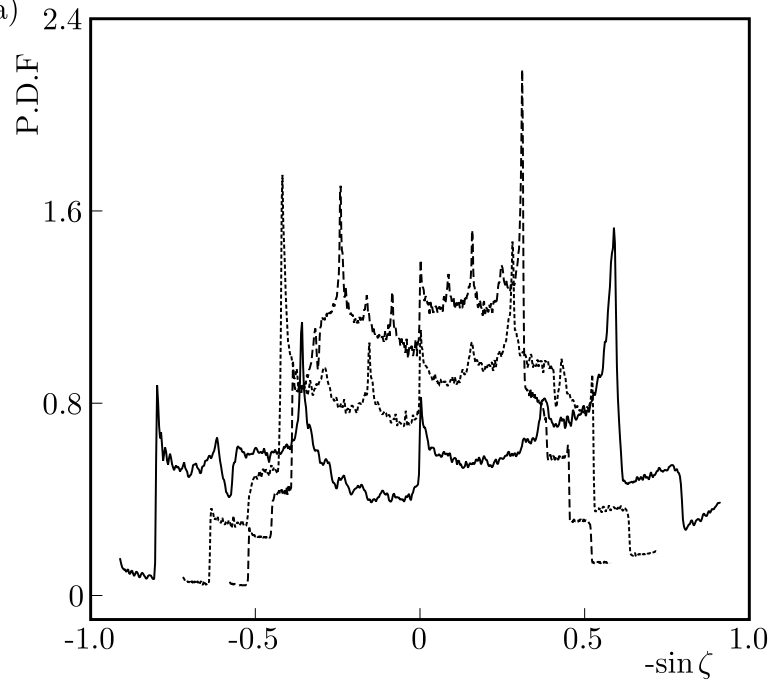

(b)

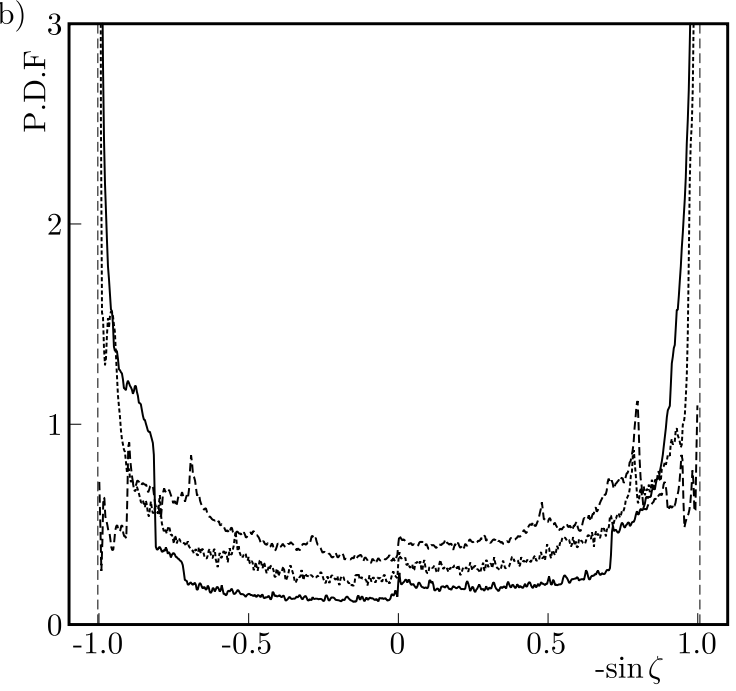

Fig. 10. P.d.f's of tracer gradient orientation; (a) $\alpha=1$, (b) $\alpha=3$; solid line: $\mathrm{St}=2$, short dash: St $=3$, long dash: $\mathrm{St}=4$

\subsection{Middle-range Strouhal number}

\subsubsection{Vanishing mean growth rate and resonance phenomenon}

Poor stirring properties are also found for Strouhal number ranges in which the mean residence time takes special values. For $\alpha=1$, the mean growth rate is virtually zero -0.021 and 0.0030 , while the maximum value is 0.88 - at $\mathrm{St}=1.5$ and $\mathrm{St}=2.5$ (Fig. 5 ) where $T_{r} / T_{f}=1.52$ and 2.52, respectively (Fig. 2). For $\alpha=3\langle\eta\rangle=0.012-$ maximum value: 0.99 - at $\mathrm{St}=3.4375$ for which $T_{r} / T_{f}=3.52$. These results are reminiscent of the resonance phenomenon found by Okkels and Tabeling (2004) in which the folding rate of an interface segment falls to zero when $T_{r} / T_{f}=(n+1 / 2)$ - with $n \in \mathbb{N}$. The authors show that a resonance occurs when the perturbation of a point on the interface developing within the first half of the mixing region is reversed and strictly offset in the second part - incidentally, this needs $n \geqslant 1$. The analysis in terms of the tracer gradient orientation agrees with this mechanism. Figure 11, plotted for $\alpha=1$ and $\mathrm{St}=1.5$, shows that the interplay between the periodic flow forcing and the position on a trajectory brings about a symmetric Lagrangian signal of strain over the residence time inside the mixing cavity.

Especially, the strain tilting events felt by the tracer gradient along a Lagrangian path are symmetric. They occur for $\sigma=0$, namely whenever $y+\alpha x \cos \omega_{f} t=0$, which is true near the center of the cavity - where $x=y=0$, but also at any position provided that $x, y$ and $t$ fulfill the latter condition - as shown in Fig. 11. The special behaviour of strain results in a symmetric signal for $\sin \zeta$ and thus for $\eta$ which, on average, cancels out over the mean residence time. This analysis is confirmed by the Lagrangian plots for $\alpha=1$ and St $=2.5$ as well as for $\alpha=3$ and $\mathrm{St}=3.4375$ (not shown). Figures 11 also displays the Lagrangian evolution of $Q$.

\subsubsection{Negative mean growth rate}

Negative values of the mean growth rate are found over narrow ranges of the Strouhal number and especially for $\alpha=1$ (Fig. 5). They obviously result from a statistical alignment of the tracer gradient closer to the extensive than to the compressive strain direction. In these special conditions, the flowfield opposes stirring which may prevent mixing. While $\langle\eta\rangle /\langle\sigma\rangle=-0.025$, at best, for $\alpha=3$, the most significant negative values of $\langle\eta\rangle$ for $\alpha=1$ are found at St $=0.1875$, 0.425 and 0.85 where $\langle\eta\rangle /\langle\sigma\rangle=-0.12,-0.11$ and -0.11 , respectively. In this ranges of the 
(a)

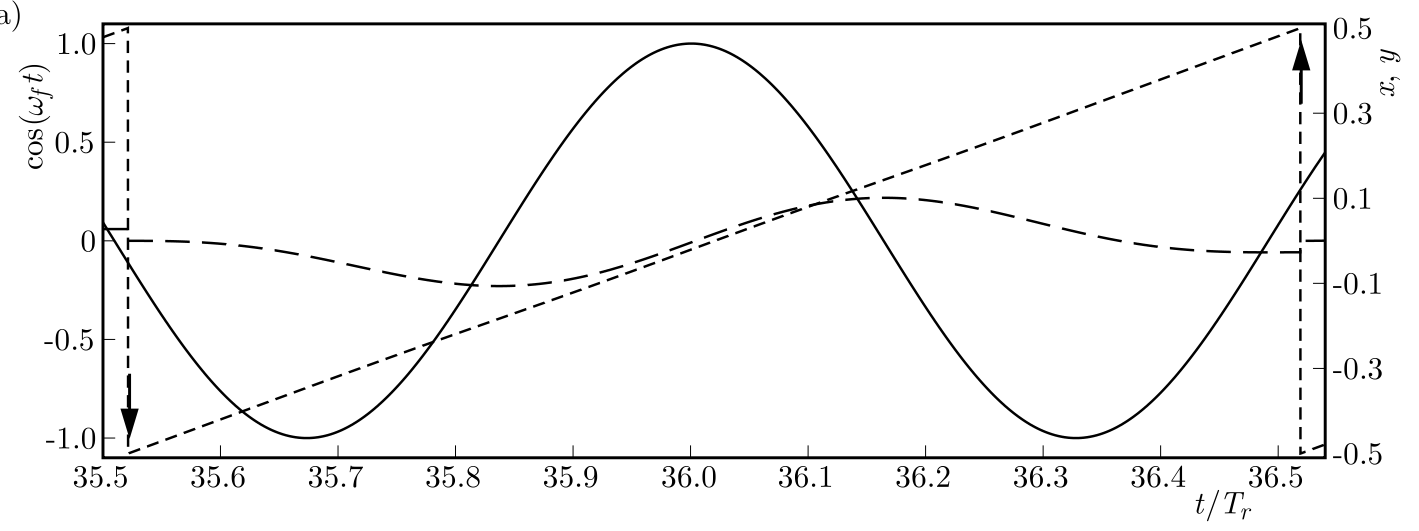

(b)

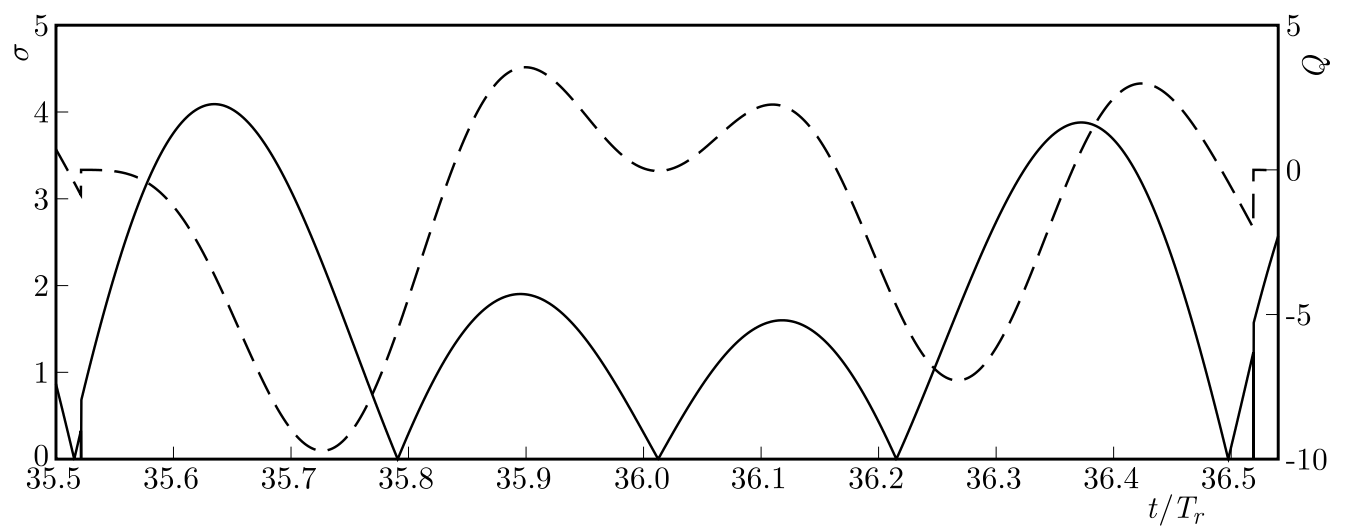

(c)

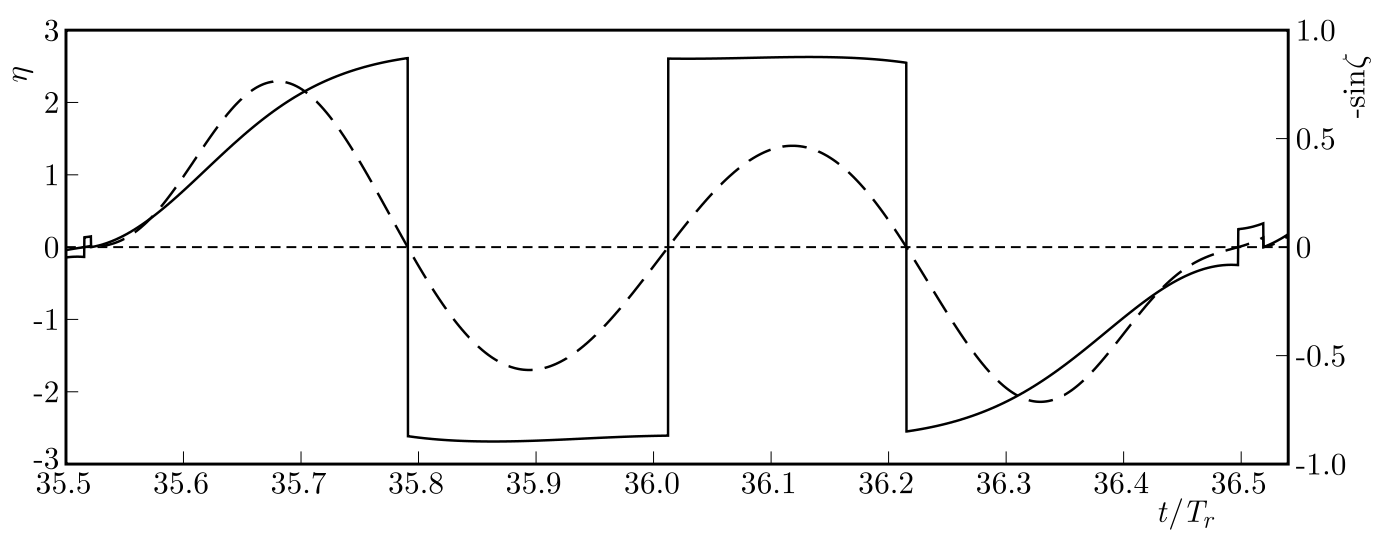

Fig. 11. Lagrangian signals for $\alpha=1$ and $\mathrm{St}=1.5$; (a) solid line: $\cos \omega_{f} t$, short dash: $x$, long dash: $y$; the arrows show the injection and exit of the Lagrangian particle; (b) solid line: $\sigma$, dashed line: $Q$;

(c) solid line: $-\sin \zeta$, dashed line: $\eta$

Strouhal number, $T_{r} / T_{f}$ takes special values $-\sim 0.5$ for $\mathrm{St}=0.1875$ and $0.425, \sim 1$ for $\mathrm{St}=0.85$ (Fig. 2). At $\mathrm{St}=0.1875$, the tracer gradient evolution is mainly governed by shear (Section 5.1). At $\mathrm{St}=0.425$ and 0.85 , both the flow structure and orientation dynamics explain the negative values of the mean growth rate. As shown in Fig. 3, the ratio of rotation events sharply peaks near these St values and the tracer gradient essentially experiences an elliptic regime. And the sharp drops of $-\sin \zeta$ at $\mathrm{St}=0.425$ and 0.85 (Fig. 12), showing statistical alignment near the extensive strain direction, deepen negative $\langle\eta\rangle$.

At those Strouhal numbers, this statistical alignment with extensive strain in hyperbolic regions is explained by unsteadiness. In fact, the time spent in the hyperbolic regime is much too short for strain to bring back the tracer gradient from the extensive to the compressive direction after tiltings occuring in the elliptic regions. 


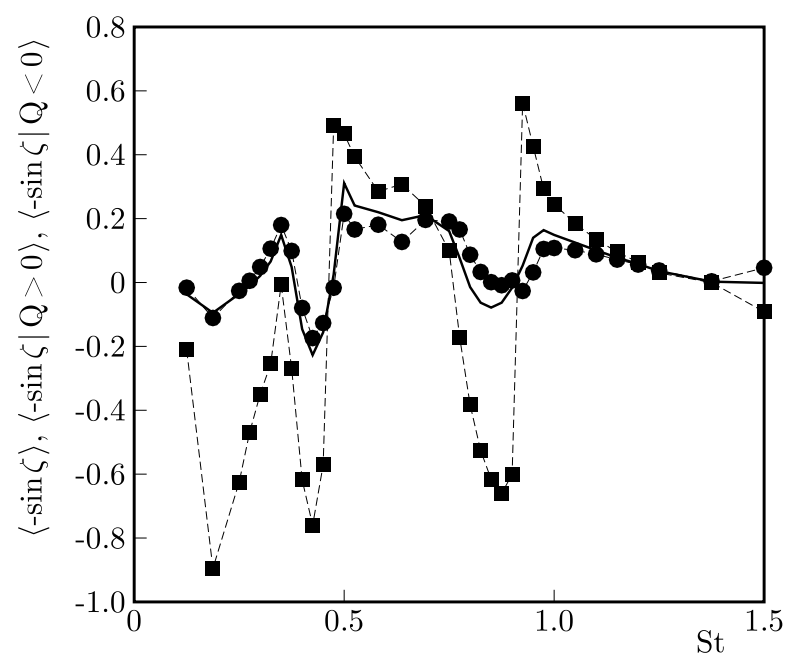

Fig. 12. Statistical orientation of the tracer gradient; $\alpha=1$; solid line: $\langle-\sin \zeta\rangle$, squares: conditioning on hyperbolic events, $\langle-\sin \zeta \mid Q>0\rangle$, circles: conditioning on elliptic events, $\langle-\sin \zeta \mid Q<0\rangle$

\subsubsection{Maximum mean growth rate}

Finally, good stirring must be restricted to moderate Strouhal numbers away from the small and large Strouhal number ranges where the tilting of strain principal axes make the mean growth rate collapse (Sections 5.1 and 5.2). In addition, $T_{r} / T_{f}$ must not take special values resulting in limiting mechanisms such as resonances. Then, good stirring conditions are found, roughly, from St $=0.48$ to 0.75 for $\alpha=1$ and St $=0.95$ to 1.6 for $\alpha=3$ (Figs. 4 and 5 ) with peak values at $\mathrm{St}=0.5$ and 1.25 , respectively. In a pure kinematic view, these findings are consistent. The ratio of strain-dominated events as well as the mean strain rate indeed reach maximum values over those Strouhal number ranges (Figs. 3 and 5). And the maximum of $\langle-\sin \zeta\rangle$ agrees with the peak values of $\langle\eta\rangle$. As already mentioned by Lee et al. (2007), optimal stirring is thus ensured by the values of the control parameters, St and $\alpha$, defining the best conditions for particles to reach a hyperbolic region and spend enough time therein. Figure 13 shows the Lagrangian signals for $\alpha=1$ and $\mathrm{St}=0.5$.

By $x=$ cte-segments, the trajectories inside the cavity reveal the inroads into the side channels. As a possible result of the chaotic behaviour, they do not display any clear periodic features. Instead, it appears that a particle may either cross the mixing zone straight or spend a variable time inside the side channel. The sample signals also confirm the significant ratio of strain-dominated events as well as the relative large time spent by the tracer gradient near the compressive direction of strain.

\section{Conclusion}

This study confirms that despite the significant role of the strain level, the response of the tracer gradient orientation to unsteady strain is a key mechanism to explain the stirring properties of a periodically forced, cross-channel micromixer.

This approach also gives a detailed insight into the micromixer properties. At small Strouhal numbers, the mean growth rate of the tracer gradient shrinks, thus showing poor stirring; the major part of the mean residence time is spent in the side channels where the periodic, symmetric reversing of the orientation of the tracer gradient with respect to the strain principal axes cancels its growth rate. At large Strouhal numbers, the tracer gradient does not respond to the fast 
(a)

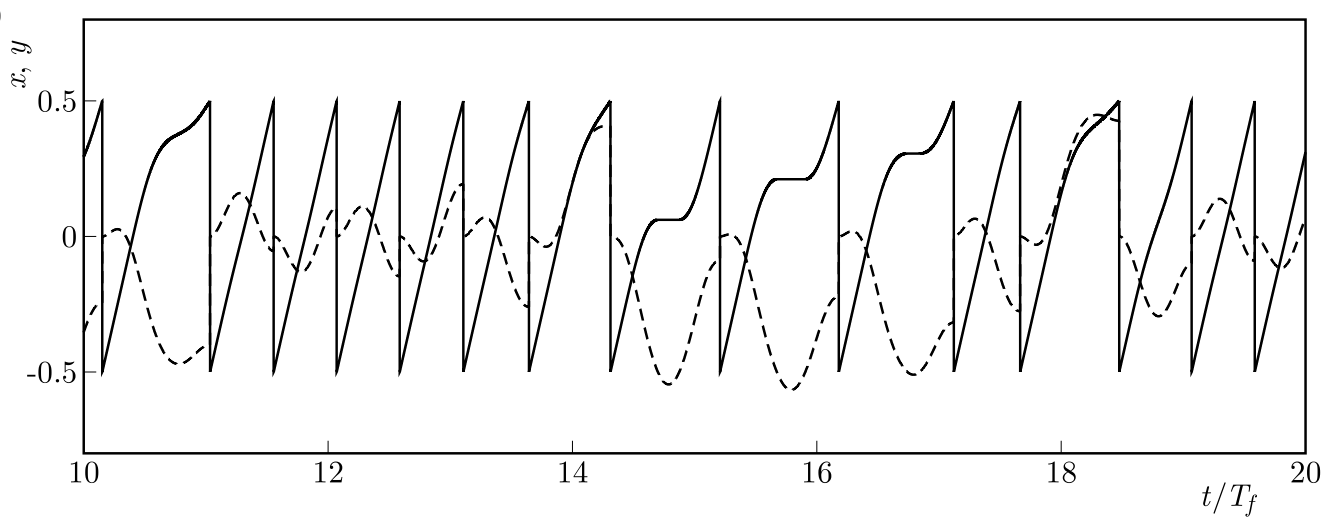

(b)

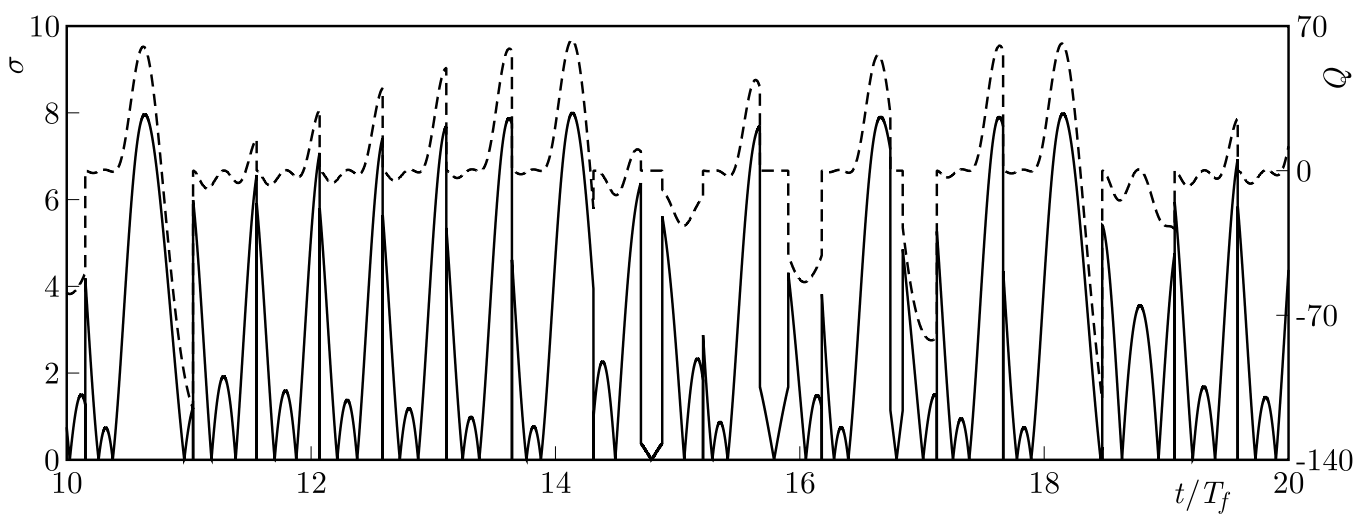

(c)

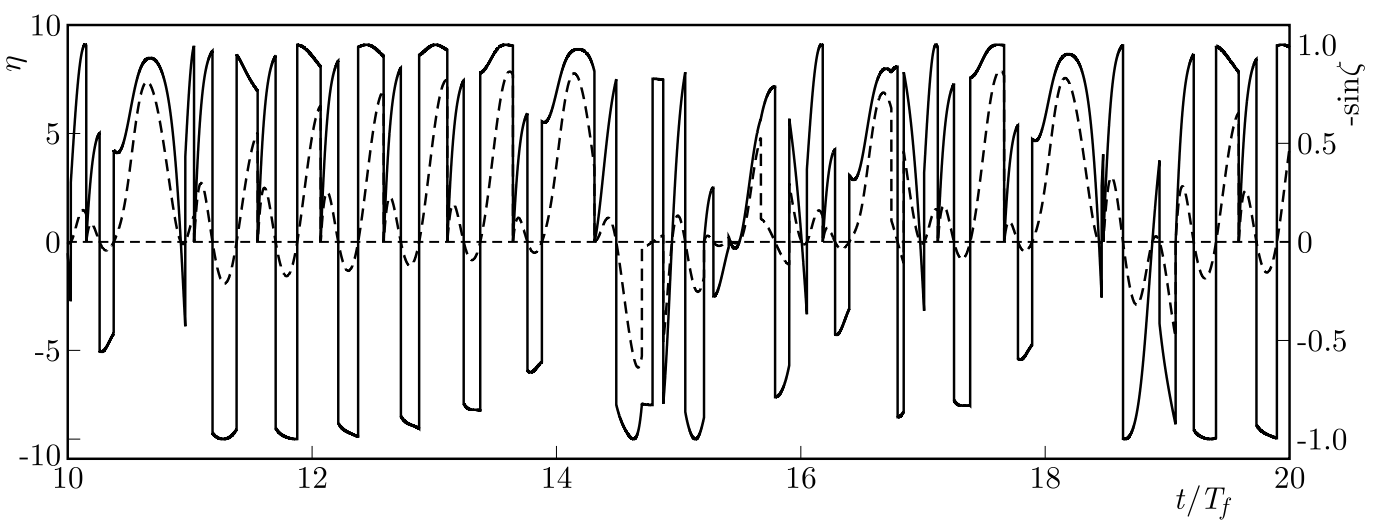

Fig. 13. Lagrangian signals for $\alpha=1$ and $\mathrm{St}=0.5$; (a) solid line: $x$, dashed line: $y$, (b) solid line: $\sigma$, dashed line: $Q ;(\mathrm{c})$ solid line: $-\sin \zeta$, dashed line: $\eta$

changes in the strain direction caused by flow forcing and aligns closer and closer to a bisector of strain principal axes where the growth rate vanishes.

Good stirring conditions are found in the middle range of the Strouhal number. They are fulfilled at Strouhal numbers for which the hyperbolic regime prevails and the mean strain rate reaches its maximum values on Lagrangian trajectories, namely within $\mathrm{St}=0.48$ to 0.75 for $\alpha=1$ and $\mathrm{St}=0.95$ to 1.6 for $\alpha=3$ with peak values at $\mathrm{St}=0.5$ and 1.25 , respectively. Poor stirring, however, may occur in narrow windows of the Strouhal number where the mean growth rate either vanishes - which precisely corresponds to the resonance phenomenon pointed out by Okkels and Tabeling (2004) - or takes negative values as a result of both the flow structure and dynamics of tracer gradient orientation when the elliptic regime prevails along the Lagrangian trajectories. 


\section{References}

1. Capretto L., Cheng W., Hill M., Zhang X., 2011, Micromixing within microfluidic devices, Topics in Current Chemistry, 304, 27-68

2. Garcia A., Gonzalez M., Paranthoën P., 2005, On the alignment dynamics of a passive scalar gradient in a two-dimensional flow, Physics of Fluids, 17, 117102

3. Garcia A., Gonzalez M., Paranthö̈n P., 2008, Nonstationary aspects of passive scalar gradient behaviour, European Journal of Mechanics B/Fluids, 27, 433-443

4. Gonzalez M., Paranthö̈n P., 2010, On the role of unsteady forcing of tracer gradient in local stirring, European Journal of Mechanics B/Fluids, 29, 143-152

5. Lapeyre G., HuA B.L., Klein P., 2001, Dynamics of the orientation of active and passive scalars in two-dimensional turbulence, Physics of Fluids, 13, 251-264

6. Lapeyre G., Klein P., Hua B.L., 1999, Does the tracer gradient vector align with the strain eigenvectors in 2D turbulence?, Physics of Fluids, 11, 3729-3737

7. Lee C.-Y., Chang C.-L., Wang Y.-N., Fu L.-M., 2011, Microfluidic mixing: a review, International Journal of Molecular Sciences, 12, 3263-3287

8. Lee Y.-K., Shin C., Tabeling P., Ho C.-M., 2007, Experimental study and nonlinear dynamic analysis of time-periodic micro chaotic mixers, Journal of Fluid Mechanics, 575, 425-448

9. NGuyen N.-T., Wu Z., 2005, Micromixers - a review, Journal of Micromechanics and Microengineering, 15, R1-R16

10. NiU X., LeE Y.-K., 2003, Efficient spatial-temporal chaotic mixing in microchannels, Journal of Micromechanics and Microengineering, 13, 454-462

11. Okkels F., Tabeling P., 2004, Spatiotemporal resonances in mixing of open viscous fluids, Physical Review Letters, 92, 038301

12. Окuво A., 1970, Horizontal dispersion of floatable particles in the vicinity of velocity singularities such as convergences, Deep-Sea Research, 17, 445-454

13. Tabeling P., Chabert M., Dodge A., Jullien C., Okkels F., 2004, Chaotic mixing in crosschannel micromixers, Philosophical Transactions of the Royal Society of London A, 362, 987-1000

14. Weiss J., 1991, The dynamics of enstrophy transfer in two-dimensional hydrodynamics, Physica $D, 48,273-294$ 